\title{
PADRÕES DA TEMPERATURA DA SUPERFÍCIE DO OCEANO ATLÂNTICO NORTE E A CONVECÇÃO DE VERÃO SOBRE A AMÉRICA DO SUL - ANÁLISE OBSERVACIONAL
}

\author{
Rosane Rodrigues Chaves \\ Recebido em 17 janeiro, 2005 / Aceito em 20 julho, 2005 \\ Received on January 17, 2005 / Accepted on July 20, 2005
}

\begin{abstract}
The association between sea surface temperature (SST) over North Atlantic Ocean and summer convection over South America and adjacent areas is studied using the principal components analysis, singular value decomposition (SVD) analysis and linear correlation. Only third mode of variability of the SST anomalies over North Atlantic Ocean is associated with summer convection over South America. It explains $9.4 \%$ of the variance. The results show that the positive (negative) SST anomalies in the North Atlantic Equatorial and subtropical (tropical) are associated with the summer convecction over the northernmost region of the South America.
\end{abstract}

Keywords: North Atlantic, South America, SST, convection.

RESUMO. Os modos dominantes das anomalias não normalizadas de Temperatura da Superfície do Mar (TSM) do Oceano Atlântico Norte (Equador e $40^{\circ} \mathrm{N} \mathrm{e} 70^{\circ} \mathrm{W}$ a $0^{\circ}$ ) são determinados através da Análise de Componentes Principais para o período de 1979 a 2001. A relação entre estes modos e a convecção sobre a América do Sul é determinada através da correlação entre as séries temporais destes e as anomalias não normalizadas de Radiação de Onda Longa (ROL). Os modos de acoplamentos das anomalias normalizadas de TSM do Atlântico Norte com as anomalias não normalizadas de ROL sobre a América do Sul e áreas adjacentes são determinados através da Decomposição em Valores Singulares (SVD) para dezembro-janeiro-fevereiro (DJF) de 1979 a 2001. Apenas o terceiro modo de variabilidades das anomalias de TSM do Atlântico Norte apresenta associação com significância estatística com a convecção sobre a América do Sul. Este modo explica 9,4\% da variância das anomalias de TSM sobre esta bacia. Neste modo, a convecção no setor norte da América do Sul está associada com anomalias positivas (negativas) de TSM na região equatorial e subtropical (tropical) desta bacia. 0 campo de TSM associado com o principal modo de acoplamento entre as anomalias de TSM do Atlântico Norte e de anomalias de ROL sobre a América do Sul corresponde ao terceiro modo de variabilidade das anomalias de TSM sobre o Atlântico Norte. Ou seja, aparentemente o terceiro modo de variabilidade das anomalias de TSM sobre o Atlântico Norte, que parece intrínseco a esta Bacia, tem maior influência sobre a convecção da América do Sul em DJF que os dois primeiros modos. Os resultados da análise observacional da TSM do Atlântico Norte com as anomalias de ROL sobre a América do Sul indicam que a TSM desta Bacia tem influência apenas sobre a convecção na região norte deste continente nos meses de DJF. Assim, os resultados apresentados neste artigo indicam que a previsibilidade da convecção sobre maior parte da América Sul nos meses de verão tem pouca dependência das condições da TSM sobre área do Atlântico Norte aqui considerada.

Palavras-chave: Atlântico Norte, América do Sul, TSM, convecção.

UENF - Universidade Estadual do Norte Fluminense Darcy Ribeiro, LENEP - Laboratório de Engenharia e Exploração de Petróleo, Rod. Amaral Peixoto, Km 163, Av. Brenand s/n - 27925-310 Imboacica, Macaé, RJ - Telefax: 2227969700 - E-mail: rosane@lenep.uenf.br 


\section{INTRODUÇÃo}

Sabe-se da importância das anomalias de Temperatura da Superfície do Mar (TSM) do Atlântico Norte para a convecção do norte do Nordeste Brasileiro (NEB; Hastenrath \& Heller, 1977; Moura \& Shukla, 1981; Nobre \& Shukla, 1996). A estação chuvosa desta região em março-abril-maio (MAM) está associada com menor aquecimento desta bacia e conseqüentemente deslocamento meridional da Zona de Convergência Intertropical (ZCIT) para sul. Já a influência da variabilidade da TSM do Atlântico Sul na convecção de verão sobre a América do Sul começa a despertar interesse da comunidade científica como em Robertson \& Mechoso (2000), Barros et al. (2000), Doyle \& Barros (2002) e Chaves \& Nobre (2004). No entanto, existem poucos trabalhos avaliando a importância das anomalias de TSM do Atlântico Norte na convecção de verão da América do Sul.

Alguns autores têm estudado a influência do Oceano Atlântico tropical sobre a precipitação da região Amazônica equatorial. Fu et al. (2001) através de experimentos numéricos mostram que a TSM do Atlântico tropical exerce influência na precipitação da parte leste desta região nas estações equinociais e nas estações de solstício esta influência é reduzida. Liebmann \& Marengo (2001) verificam que a TSM do Atlântico Equatorial está positivamente correlacionada com a precipitação do leste da Amazônia durante 0 outono e a primavera. Segundo estes autores a precipitação de dezembro-janeiro-fevereiro (DJF) nesta região não apresenta correlação significativa com a TSM do Atlântico equatorial. Buchmann et al. (1990) verificam através de experimentos numéricos que as secas na parte central da América do Sul no verão de 1986 e 1988 estão associadas ao aquecimento anômalo da região tropical norte e sul do Atlântico, com precipitação abaixo (acima) da média sobre o NEB quando este aquecimento encontra-se ao norte (sul) do equador.

Enquanto os artigos citados acima consideram o Atlântico tropical, aqui neste artigo considera-se apenas a região do Atlântico Norte entre o Equador e $40^{\circ} \mathrm{N}$. Este procedimento é feito para avaliar separadamente a influência do setor norte do Atlântico sobre a convecção de verão da América do Sul e também como forma de eliminar o problema da correlação interhemisférica artificial que uma análise envolvendo a parte norte e sul desta Bacia pode ocasionar (Houghton \& Tourre, 1992, Dommenget \& Latif, 2000). Aqui são determinados os principais modos das anomalias não normalizadas de TSM no Atlântico Norte através da Análise de Componentes Principais (ACP) e sua associação com a convecção da América do Sul. Como também, são determinados os modos de acoplamentos entre as anomalias normalizadas de TSM do Atlântico Norte com as anomalias não normalizadas de Radiação de Onda Longa (ROL) sobre a América do Sul e áreas adjacentes através da Decomposição em Valores Singulares (SVD).

\section{DADOS E METODOLOGIA}

Neste trabalho são utilizados os campos mensais de TSM do Comprehensive Ocean-Atmosphere Data Set (COADS) Wolter (1997) e dados de ROL do National Oceanic and Atmospheric Administration/Coorperative Institute for Research in Enviromment Science (NOAA-CIRES) Climate Diagnostics Center (CDC), Boulder, Colorado, USA Liebmann e Smith (1996).

0 COADS é o conjunto de dados oceânicos mais completo e mais longo disponível atualmente para pesquisa sobre clima global (Wolter, 1997) e estão disponíveis a partir de 1854 até 0 mês atual. Os dados do COADS contêm observações principalmente de navios, no entanto, nos últimos dez anos tem havido contribuição dos dados de satélite. Antes de ser disponibilizada à comunidade científica a qualidade destes dados é avaliada por métodos estatísticos (Wolter, 1997). Estes dados estão disponíveis na "home-page" do CDC (http://www.cdc.noaa.gov).

Os campos de ROL também foram obtidos da "home-page" do CDC. Estes dados provêm do satélite NOAA e estão disponíveis a partir de junho de 1974 até os dias atuais para todo o globo, com resolução de $2,5^{\circ}$ latitude por $2,5^{\circ}$ Iongitude. As falhas destes dados são preenchidas através de um processo de interpolação espacial e temporal no CDC Liebmann e Smith (1996) e representam a média diária dos horários das 12 e 00UTC. A ROL medida por satélite pode ser usada como uma aproximação para a convecção profunda nos trópicos e nos subtrópicos durante 0 período de verão, uma vez que valores baixos de ROL sobre estas regiões geralmente indicam atividade convectiva e também cobertura de nuvens cirrus associada à convecção profunda (Zhang, 1993).

A ACP é uma técnica estatística de análise multivariada baseada fundamentalmente em operações de matrizes. Esta técnica é útil para identificação objetiva dos modos de variabilidade de observações atmosféricas e oceânicas (Kutzbach, 1967; Ward \& Folland, 1991; Kousky \& Kayano, 1994). 0 principal objetivo da ACP é gerar um conjunto reduzido de dados que pode representar a variabilidade temporal dos padrões espaciais que explica a maior fração da variância da série original. 0 método possibilita também a análise de dependência entre as diversas variáveis, ou ainda entre as diversas regiões do domínio de interesse. Detalhes deste método podem ser obtidos em Richman (1986), Wallace et al. (1992), Wilks (1995) e von Storch \& Navarra (1999). 
Neste trabalho a ACP é empregada para determinar os modos dominantes das anomalias não normalizadas de TSM no Atlântico Norte (Equador e $40^{\circ} \mathrm{Ne} 70^{\circ} \mathrm{W}$ a $0^{\circ}$ ) através da matriz de correlação dos dados de anomalia não normalizada de TSM para o período de 1979 a 2001. Posteriormente, a relação destes modos com a convecção é determinada através da correlação linear entre as séries temporais destes modos e as anomalias não normalizadas de ROL entre $60^{\circ} \mathrm{S}$ a $60^{\circ} \mathrm{N}$.

Enquanto a ACP fornece uma base ortogonal para um determinado conjunto de dados que maximiza a variância explicada pelo mesmo, a análise de Decomposição em Valores Singulares tem como objetivo identificar padrões espaciais de acoplamento que maximizam a covariância explicada por dois ou mais conjuntos de dados. Ao contrário da ACP, a análise de SVD pode ser aplicada em matrizes não-quadráticas e não-simétricas e estes dois conjuntos de dados não necessariamente precisam conter o mesmo número de pontos de grade. A análise de SVD tem sido empregada para investigar a associação entre a circulação atmosférica e a TSM (Wallace et al., 1992), como também 0 impacto da TSM sobre a precipitação regional (Coelho et al., 2002). Este método também tem se mostrado útil na investigação da interação oceano-atmosfera no Pacífico (Wallace et al., 1992), no Atlântico Norte (Peng \& Robinson, 2001) e no Atlântico Sul (Venegas et al., 1997).

Os modos de acoplamentos das anomalias normalizadas de TSM do Atlântico Norte (Equador e $40^{\circ} \mathrm{N}$ e $70^{\circ} \mathrm{W}$ a $0^{\circ}$ ) com as anomalias não normalizadas de ROL sobre a América do Sul e áreas adjacentes $\left(40^{\circ} \mathrm{S}\right.$ a $20^{\circ} \mathrm{N} \mathrm{e} 90^{\circ} \mathrm{W}$ a $\left.20^{\circ} \mathrm{W}\right)$ são determinados aplicando-se a análise de SVD na matriz de covariância destes dados para os meses de DJF de 1979 a 2000. Para melhor avaliar estes modos de acoplamento, as séries temporais correspondentes aos dados de anomalia de TSM e de ROL obtidas através da análise de SVD são correlacionadas com as anomalias de TSM entre $40^{\circ} \mathrm{S}$ e $40^{\circ} \mathrm{N}$.

0 campo de correlação da série temporal das anomalias de TSM (ROL), determinado através de SVD, com o campo das anomalias de TSM (ROL) é chamado de campo homogêneo das anomalias de TSM (ROL). Enquanto que o campo de correlação da série temporal das anomalias de ROL (TSM) com o campo das anomalias de TSM (ROL) é chamado de campo heterogêneo das anomalias de TSM (ROL). Este procedimento permite fazer uma melhor avaliação dos padrões de acoplamento, uma vez que os campos heterogêneos mostram mais diretamente a relação entre os dois campos (Trenberth et al. 2002). Aqui por exemplo, permite avaliar a melhor a influência das anomalias de TSM do Atlântico Norte sobre a convecção da América Sul, uma vez que a influência de outros modos de variabilidade, como por exemplo, 0 El-Niño/Oscilação Sul (ENSO), também pode estar embutido nos resultados desta análise de SVD.

\section{RESULTADOS}

A Figura 1 mostra a distribuição espacial dos três primeiros autovetores das anomalias de TSM sobre 0 Atlântico Norte (Equador a $40^{\circ} \mathrm{N}$ e $70^{\circ} \mathrm{W}$ a $0^{\circ}$ ) que explicam $28,4 \%, 16,3 \%$ e $9,4 \%$ da variância, respectivamente, para o período de 1979 a 2001. A distribuição espacial do primeiro autovetor mostra um padrão monopolo, com a maior variabilidade das anomalias de TSM na região equatorial e tropical da Bacia (Fig. 1a). No segundo padrão, as anomalias de TSM da região tropical apresentam sinal oposto em relação às anomalias da região subtropical, com maior variabilidade da TSM nesta última região (Fig. 1b). 0 terceiro padrão apresenta três centros de ação sobre o Atlântico Norte, com anomalias de mesmo sinal na região equatorial e subtropical e anomalias de sinal inverso na região intermediária (Fig. 1C).

Aparentemente, a alta variabilidade interanual das séries temporais destes padrões não está associada ao fenômeno ENSO, uma vez que não são verificadas amplitudes acentuadas nestas séries em anos de eventos intensos como em 1982/83 e 1997/98 (Fig. 2). Os periodogramas destas séries mostram que os dois primeiros modos apresentam variabilidade decadal (Fig. 3a, b), no primeiro modo as freqüências dominantes são 11,6 e 4,6 anos e no segundo modo 11,6 anos. Enquanto no terceiro modo não se verifica uma freqüência dominante (Fig. 3c).

0 campo de correlação das séries temporais destes modos com as anomalias de TSM de outras áreas oceânicas indica que os dois primeiros modos podem estar associados com a variabilidade decadal da TSM nas regiões subtropicais (Fig. 4 a, b), especialmente no Pacífico Norte (Mantua et al., 1997). 0 segundo padrão das anomalias de TSM sobre o Atlântico Norte também está associado com a variabilidade da TSM da região equatorial do Pacífico (Fig. 4b). Enquanto o terceiro modo parece ser intrínseco ao Atlântico Norte (Fig. 4c), não se observando correlações significativas com as anomalias de TSM de outras regiões.

Segundo Klein et al. (1999), Saravanan \& Chang (2000) e Lau \& Nath (2000), existe conexão entre 0 ENSO e 0 Atlântico Norte. Esta associação se dá através da redução da nebulosidade devido à intensificação do ramo descendente da circulação anômala de Walker e das teleconexões atmosféricas como o Pacific - North Atlantic (PNA), resultando no aquecimento do Atlântico Norte subtropical através do aumento do fluxo de radiação de onda 

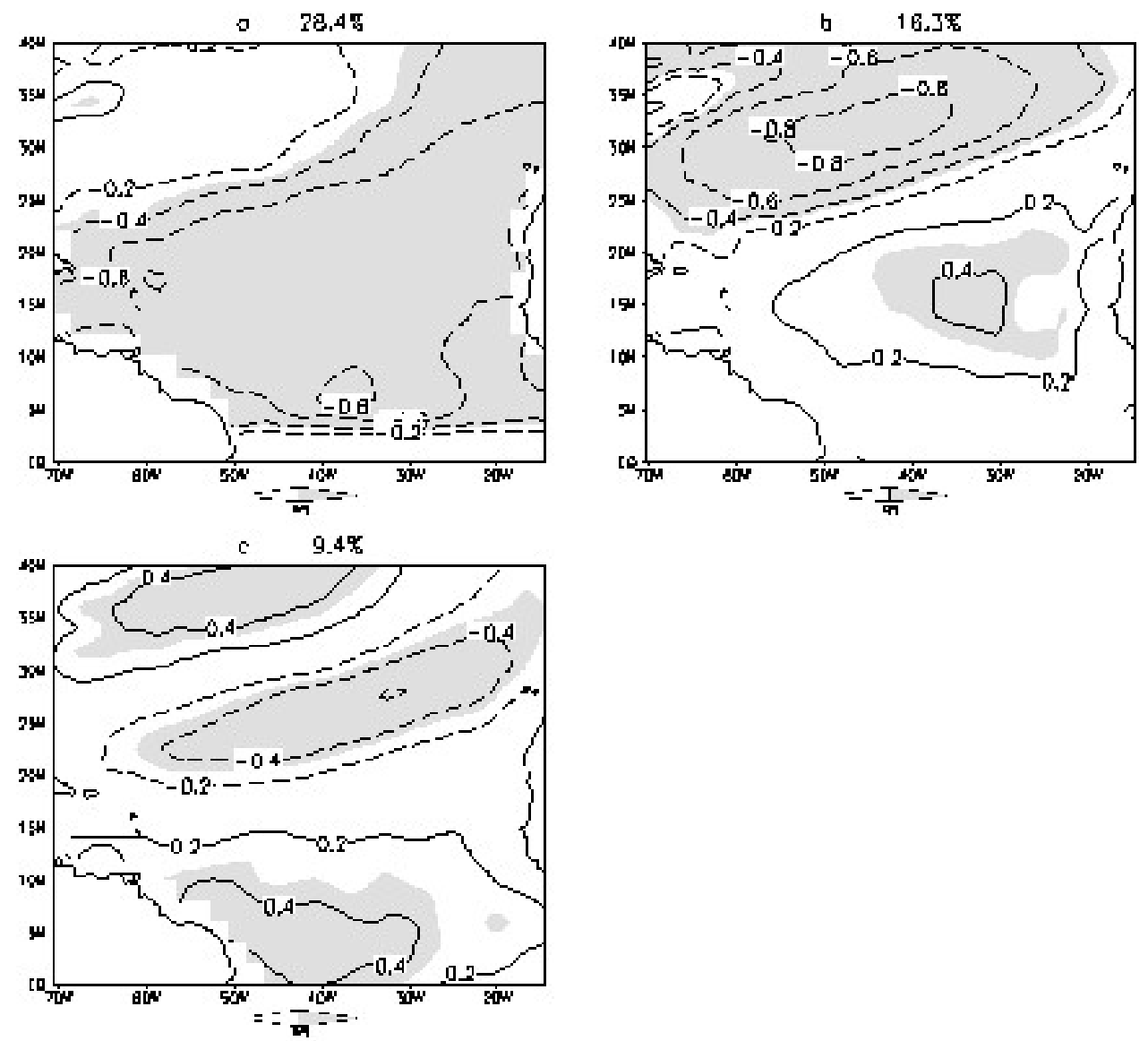

Figura 1 - Padrão espacial dos três primeiros autovetores das anomalias não normalizadas da TSM sobre 0 Oceano Atlântico Norte (Equador a $40^{\circ} \mathrm{N}$ e $70^{\circ} \mathrm{W} \mathrm{a} 0^{\circ}$ ) para o período 1979 a 2001. 0 tom de cinza representa valores com nível de significância estatística acima de $95 \%$ segundo $0 t$-Student's.

curta. Porém, para o período e a região do Atlântico Norte considerados esta associação não parece ser muito significativa.

\section{Associação entre os principais modos de variabilidade das anomalias de TSM sobre o Atlântico Norte e a convecção sobre a América do Sul}

Nesta secção considera-se somente a influência do primeiro e terceiro modo das anomalias de TSM sobre o Atlântico Norte na convecção da América do Sul, uma vez que estes apresentam acentuada variabilidade interanual. Enquanto 0 segundo modo apresenta predominância de variabilidade interdecadal, e esta não é a escala de interesse neste trabalho.

Os campos de correlação entre a série temporal do primeiro e terceiro modo de variabilidade das anomalias de TSM sobre 0 Atlântico Norte e as séries temporais das anomalias de ROL entre as latitudes $60^{\circ} \mathrm{S}$ a $60^{\circ} \mathrm{N}$ (Fig. 5), mostram que apenas o terceiro modo apresenta associação com significância estatística com a convecção sobre a América do Sul. Neste modo, a convecção no setor norte da América do Sul está associada com anomalias positivas (negativas) de TSM na região equatorial e subtropical (tropical; Fig. 5b).

Paegle \& Mo (2002) mostram que a influência das anomalias de TSM sobre o Atlântico Norte em DJF sobre a precipitação da América do Sul limita-se a uma pequena região do NEB. Fu et al. (2001) mostram que a TSM do Atlântico tropical exerce influência na parte leste desta região nas estações equinociais, no entanto no setor oeste e nas estações de solstício esta influência é reduzida. Segundo Liebmann \& Marengo (2001), a TSM no leste do Atlântico equatorial está positivamente correlacionada com a precipitação do leste da Amazônia durante o outono e a primavera. Já para o período de DJF esta associação não é significativa. Os resultados aqui apresentados são condizentes com os trabalhos mencionados acima. 


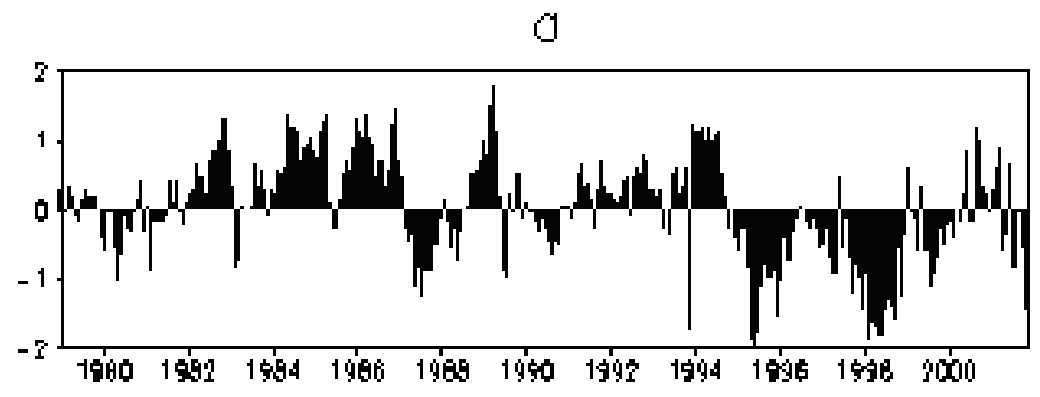

b

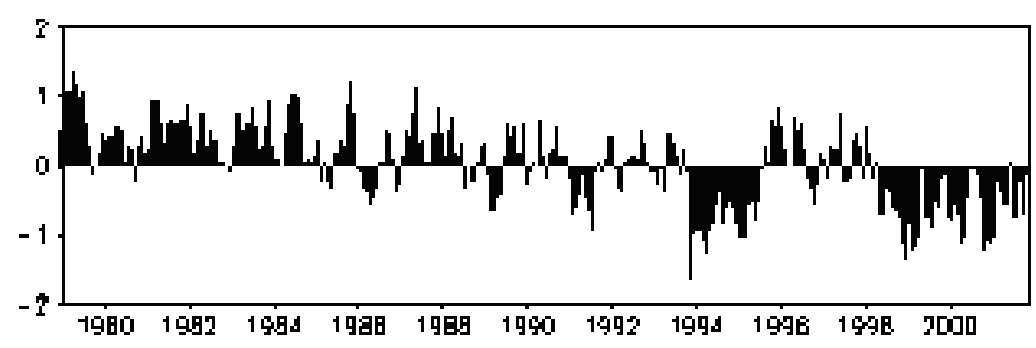

$\mathrm{C}$

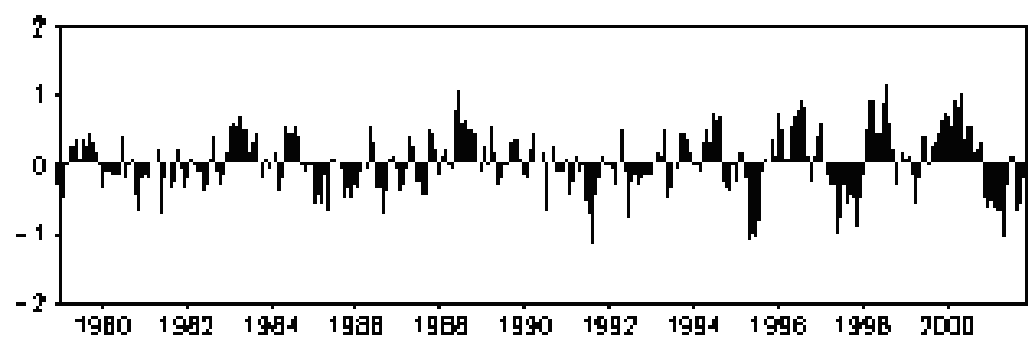

Figura 2 - Séries temporais dos três primeiros autovetores das anomalias não normalizadas de TSM sobre 0 Atlântico Norte (Equador a $40^{\circ} \mathrm{N}$ e $70^{\circ} \mathrm{W}$ a $0^{\circ}$ ) para o período de 1979 a 2001.

Modos de acoplamento entre a convecção sobre a América do Sul e áreas adjacentes e as anomalias de TSM sobre o Atlântico Norte para DJF 1979/2000

Os três principais modos de acoplamento, determinados através da SVD, entre as anomalias normalizadas de TSM sobre 0 Atlântico Norte de ROL sobre a América do Sul e áreas adjacentes (Fig. 6 e 7) para DJF de 1979 a 2000, explicam 16,7\%, 13,2\%, $7,4 \%$ da covariância entre os dois conjuntos de dados, respectivamente. Os coeficientes de correlação entre as séries temporais da SVD dos dados de anomalia de ROL e de TSM (Fig. 8), para os três primeiros modos são 0,58, 0,52 e 0,56, respectivamente. Isto indica que existe associação entre os campos acoplados destas variáveis. A Tabela 1 apresenta os coeficientes de correlação entre as séries temporais dos três primeiros modos acoplados. 0 índice de correlação entre a série temporal do primeiro e terceiro modo acoplado das anomalias de TSM (ROL) é de -0,44 $(0,48)$, mostrando que estes modos não são independentes.

Os campos homogêneos das anomalias de TSM no Atlântico Norte (Fig. 6a, b, c), mostram que o primeiro, (segundo) e [terceiro] padrão de acoplamento correspondem ao terceiro, (primeiro) [segundo] modo de variabilidade das anomalias de TSM sobre o Atlântico Norte (comparar região do Atlântico Norte da Figura 6a,b,c com a Figura 1c,a,b, respectivamente). Ou seja, 0 terceiro modo de variabilidade das anomalias de TSM do Atlântico Norte (Fig. 1c), que é um modo intrínseco a esta Bacia, parece ser mais acoplado a convecção da América do Sul e áreas adjacentes que os dois primeiros modos.

Os campos heterogêneos das anomalias de TSM (Fig. 6d, e, f), mostram que os principais modos de acoplamento das anomalias de ROL sobre a América do Sul com as anomalias de TSM 
0

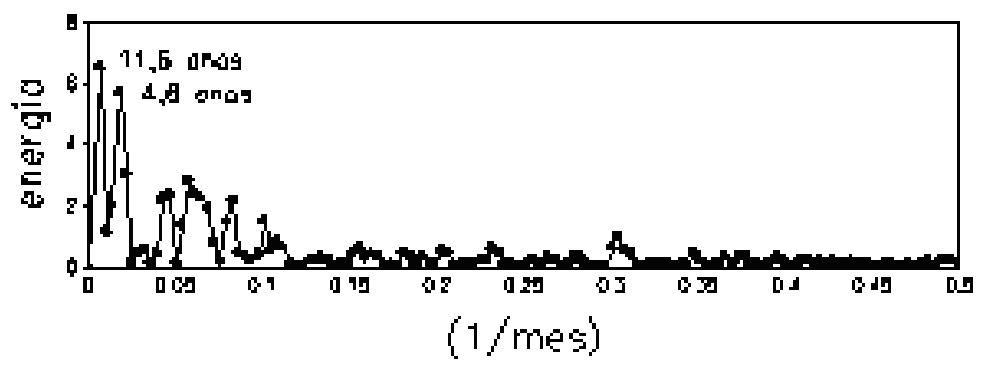

b

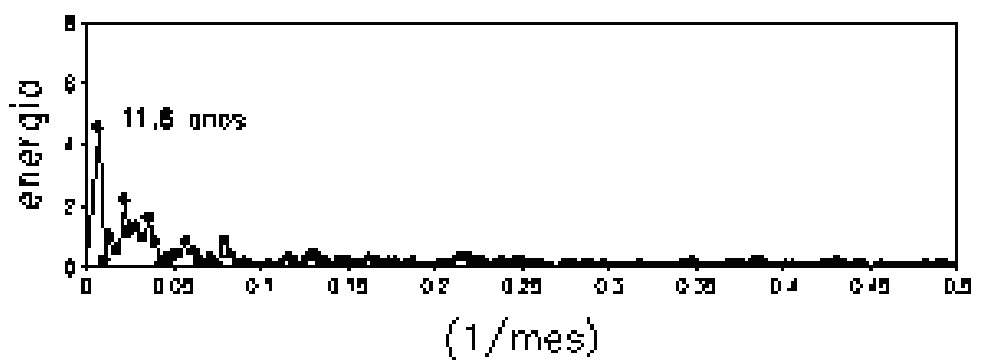

C

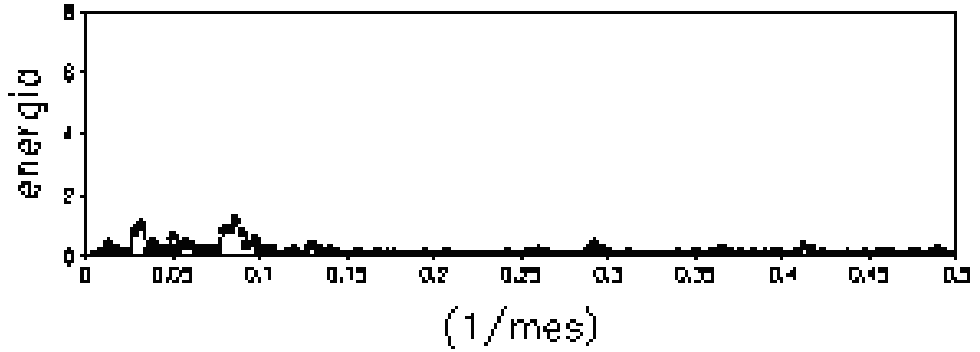

Figura 3 - Periodograma das séries temporais dos três primeiros autovetores das anomalias não normali-

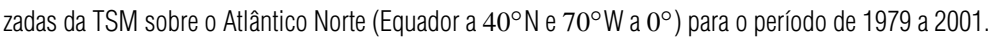

Tabela 1 - Correlação entre as séries temporais dos Valores Singulares.

\begin{tabular}{|l|c|c|c|c|c|c|}
\hline & SV1 TSM & SV2 TSM & SV3 TSM & SV1 R0L & SV2 R0L & SV3 R0L \\
\hline SV1 TSM & 1,00 & $-0,11$ & $-0,44$ & 0,58 & 0,00 & 0,00 \\
\hline SV2 TSM & & 1,00 & 0,00 & 0,00 & 0,52 & 0,00 \\
\hline SV3 TSM & & & 1,00 & 0,00 & 0,00 & 0,56 \\
\hline SV1 ROL & & & & 1,00 & 0,00 & 0,48 \\
\hline SV2 ROL & & & & & 1,00 & 0,17 \\
\hline SV3 ROL & & & & & & 1,00 \\
\hline
\end{tabular}

do Atlântico Norte estão fortemente associados à variabilidade da TSM do Pacífico equatorial.

Os campos heterogêneos de ROL (Fig. 7c, d, e), mostram que no primeiro modo acoplado a convecção acima média na costa norte da América do Sul está associada com o aquecimento do Atlântico Norte equatorial (Fig. 6a). Este resultado corrobora os obtidos no item anterior com a ACP, no qual se verifica que apenas este modo apresenta correlação estatisticamente significativa 


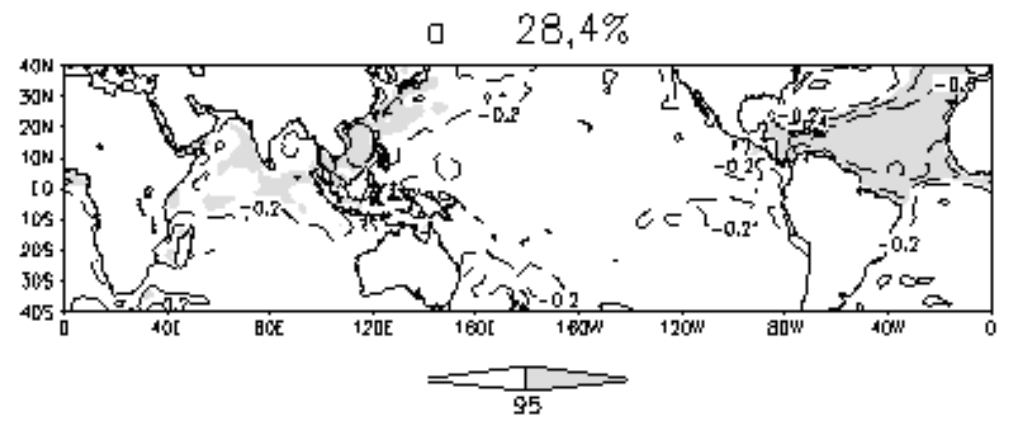

b $16,3 \%$
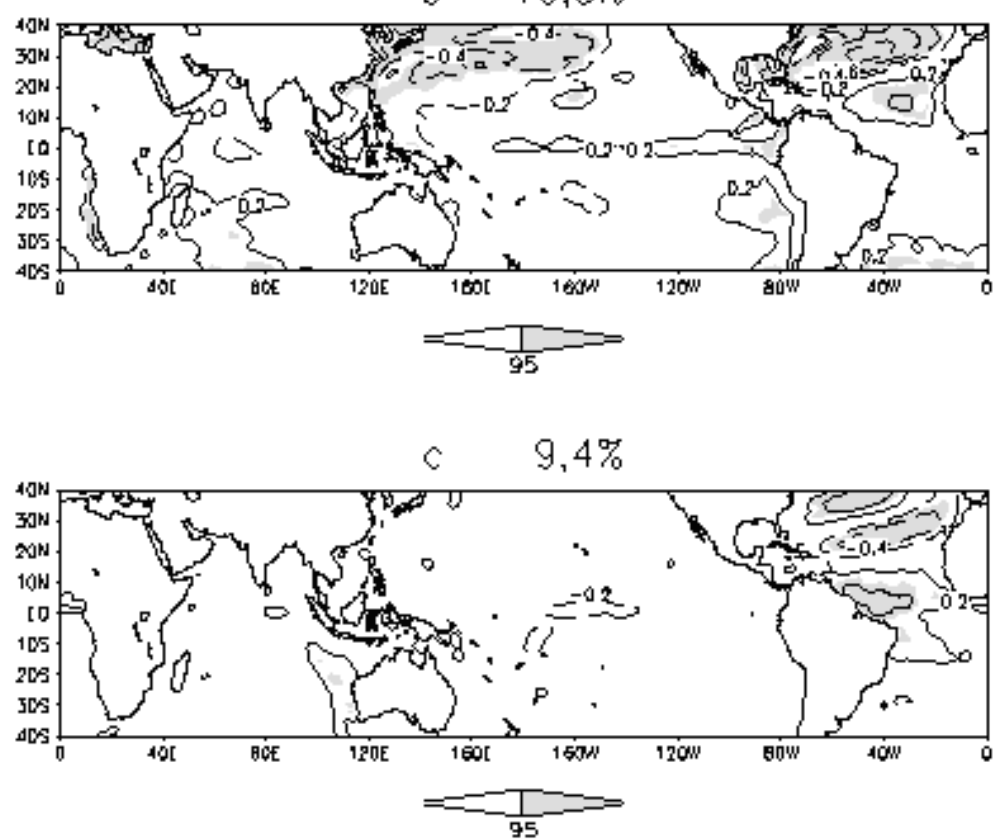

Figura 4 - Campo de correlação entre as séries temporais dos três primeiros autovetores das anomalias não normalizadas da TSM sobre o Atlântico Norte (Equador a $40^{\circ} \mathrm{N}$ e $70^{\circ} \mathrm{W}$ a $0^{\circ}$ ) e as anomalias de TSM entre $40^{\circ} \mathrm{S}$ e $40^{\circ} \mathrm{N}$ para o período de 1979 a 2001. Tom de cinza representa valores com nível de significância estatística acima de $95 \%$ segundo $t$-Student's.

com a convecção da América do Sul.

\section{CONCLUSÕES}

Os modos dominantes das anomalias não normalizadas de TSM no Atlântico Norte (Equador a $40^{\circ} \mathrm{Ne} 70^{\circ} \mathrm{W}$ a $0^{\circ}$ ) são determinados através da ACP para o período de 1979 a 2001. A relação entre estes modos e a convecção é determinada através de correlação linear entre as séries temporais destes e as anomalias não normalizadas de ROL entre $60^{\circ} \mathrm{S}$ a $60^{\circ} \mathrm{N}$. Posteriormente os modos de acoplamentos das anomalias normalizadas de TSM do Atlântico Norte com as anomalias não normalizadas de ROL sobre a América do Sul e áreas adjacentes $\left(40^{\circ} \mathrm{S}\right.$ a $20^{\circ} \mathrm{N}$ e $90^{\circ} \mathrm{W}$ a 20W) para DJF de 1979 a 2001 são determinados através da SVD.

A distribuição espacial do primeiro autovetor mostra um padrão monopolo, com a maior variabilidade das anomalias de TSM na região equatorial e tropical da Bacia. No segundo padrão, as anomalias de TSM da região tropical apresentam sinal oposto em relação às anomalias da região subtropical. 0 terceiro padrão apresenta três centros de ação sobre o Atlântico Norte, com anomalias de mesmo sinal na região equatorial e subtropical e anomalias de sinal inverso na região intermediária. 0 primeiro e terceiro modo apresentam acentuada variabilidade interanual e o segundo modo apresenta predominância de variabilidade interde- 
व

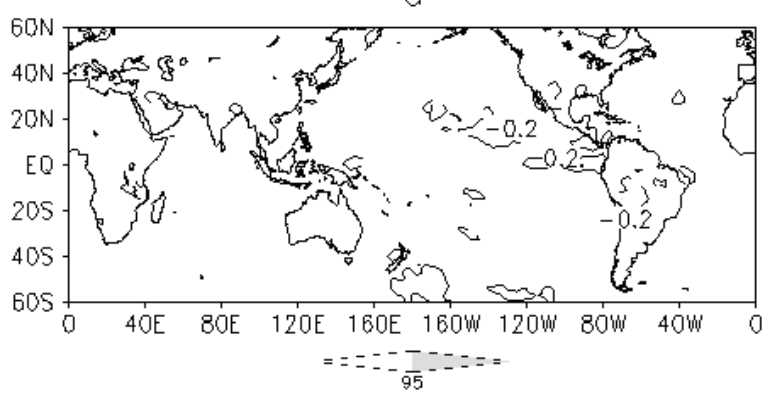

b

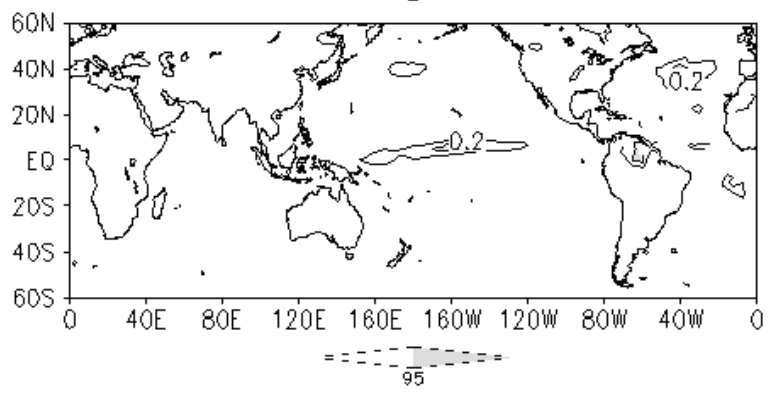

Figura 5 - Campo de correlação entre a série temporal do primeiro (a) e terceiro (b) autovetor das anomalias não normalizadas da TSM sobre o Atlântico Norte (Equador a $40^{\circ} \mathrm{Ne} 70^{\circ} \mathrm{W}$ a $0^{\circ}$ ) e as anomalias de ROL entre $60^{\circ} \mathrm{S}$ e $60^{\circ} \mathrm{N}$ para o período de 1979 a 2001. Tom de cinza representa valores com nível de significância estatística acima de $95 \%$ segundo $t$-Student's.

cadal. Apenas o terceiro modo apresenta associação com significância estatística com a convecção sobre a América do Sul. Neste modo, a convecção no setor norte da América do Sul está associada com anomalias positivas (negativas) de TSM na região equatorial e subtropical (tropical).

0 campo de TSM associado com o principal modo de acoplamento entre as anomalias de TSM do Atlântico Norte e de anomalias de ROL sobre a América do Sul corresponde ao terceiro modo de variabilidade das anomalias de TSM sobre 0 Atlântico Norte. Ou seja, aparentemente o terceiro modo de variabilidade das anomalias de TSM sobre 0 Atlântico Norte (Equador $-40^{\circ} \mathrm{N}$ ), que parece intrínseco a esta Bacia, tem maior influência sobre a convecção da América do Sul em DJF que os dois primeiros modos. Os resultados indicam que a TSM Atlântico Norte tem influência apenas sobre a convecção do setor norte da América do Sul nos meses de DJF. A convecção sobre esta região está associada com águas quente sobre a região equatorial e subtropical do Atlântico Norte, possivelmente através do transporte de umidade do oceano para a região continental.

Os resultados apresentados neste artigo indicam que a previsibilidade da convecção sobre maior parte da América Sul nos meses de verão tem pouca dependência das condições da TSM sobre 0 Atlântico Norte. No entanto, mais estudos devem ser feitos considerando esta questão, principalmente estudos envolvendo experimentos numéricos com teste de sensibilidade à TSM para a confirmação dos resultados aqui apresentados.

\section{AGRADECIMENTOS}

A autora agradece ao Dr. Prakky Satyamurty pelas sugestões. Este trabalho é parte da Tese de Doutorado da autora defendida no INPE em agosto de 2003, financiada pelo CNPq.

\section{REFERÊNCIAS}

BARROS V, GONZALEZ M, LIEBMANN B \& CAMILLONI I. 2000. Influence of the South Atlantic Convergence Zone and South Atlantic sea surface temperatures on interannual summer rainfall variability in southeastern South America. Theor. Applied Clim, 67: 123-133.

BUCHMANN J, PAEGLE J, BUJA LE \& DICKINSON RE. 1990. The effect of tropical Atlantic heating anomalies upon GCM rain forecasts over the Americas. J. Clim., 3: 189-208.

CHAVES RR \& NOBRE P. 2004. Interactions between sea surface temperature over the South Atlantic Ocean and the South Atlantic Convergence Zone. Geophys. Res. Lett. 31: L03204 doi:10.1029/2003GL018647. 

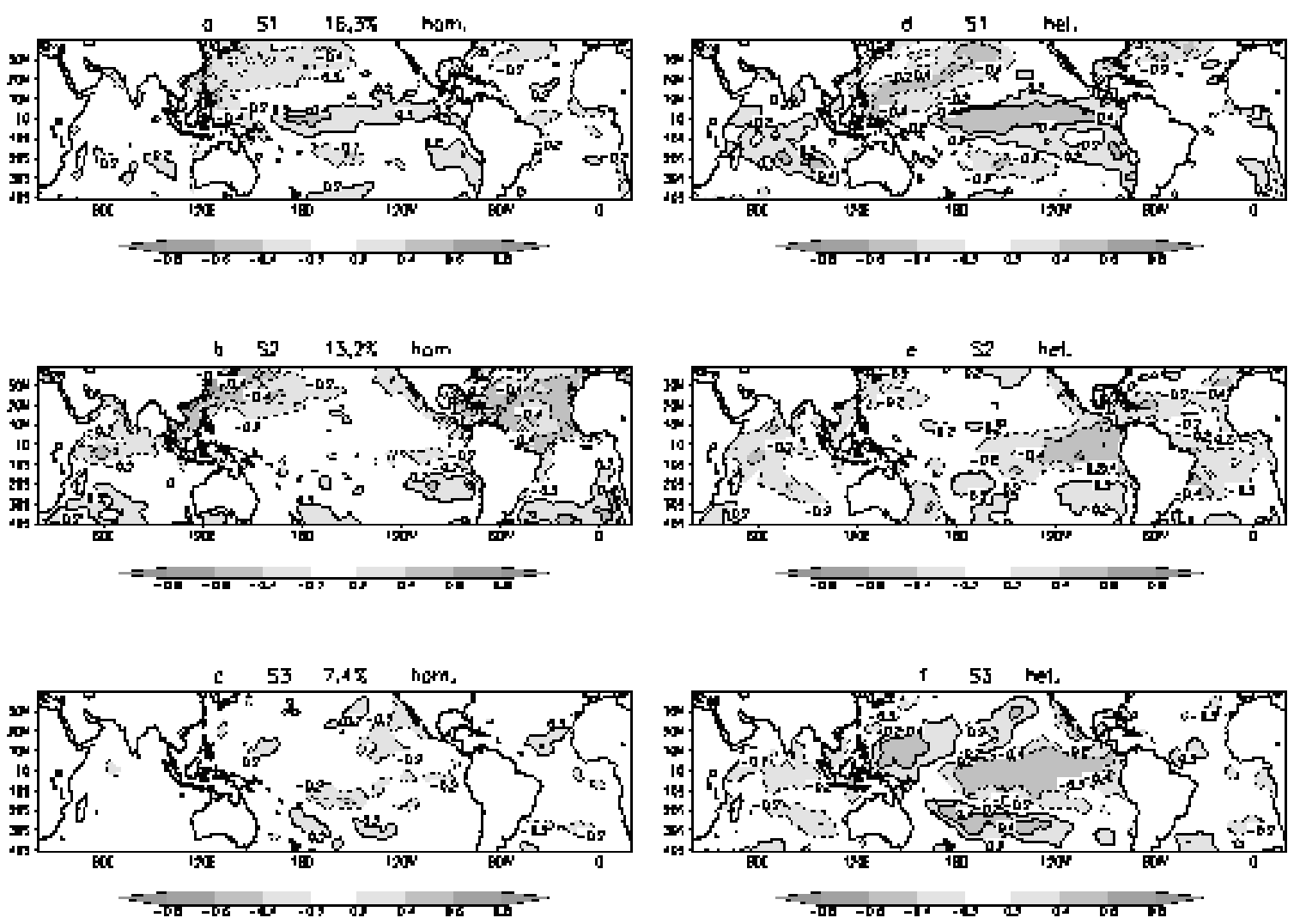

Figura 6 - Campos homogêneos (a, b, c) e heterogêneos (d, e, f) de TSM dos três primeiros modos da SVD entre as anomalias normalizadas de ROL sobre a América do Sul e de TSM sobre o Atlântico Norte para DJF de 1979/2000. Tom de cinza representa valores com nível de significância estatística acima de 95\% segundo $0 t$-Student's.

COELHO CAS, UVO CB \& AMBRIZZI T. 2002. Exploring the impacts of the tropical Pacific SST on the precipitation patterns over South America during ENSO periods. Theor. Applied Clim, 71: 185-197.

DOMMENGET D \& LATIF M. 2000. Interannual to decadal variability in the tropical Atlantic. J. Clim., 13: 777-792.

DOYLE ME \& BARROS VR. 2002. Midsummer low-level circulation and precipitation in subtropical South America and related sea surface temperature Anomalies in the South Atlantic. J. Clim., 15: 3394-410.

FU R, DICKINSON RE, CHEN M \& WANG H. 2001. Tropical sea surface temperatures influence the seasonal distribution of precipitation in the Equatorial Amazon? J. Clim., 14: 4003-4026.

HASTENRATH S \& HELLER L. 1977. Dynamics of climatic hazards in Northeast Brazil. Quart. J. Roy. Meteor. Soc., 103: 77-92.

HOUGHTON RW \& TOURRE YM. 1992. Characteristics of low-frequency sea surface temperature fluctuations in the tropical Atlantic. J. Clim., 5: 765-771.

KLEIN SA, SODEN BJ \& LAU N-C. 1999. Remote Sea Surface Temperature Variations during ENSO: Evidence for a Tropical Atmospheric Bridge. J. Clim., 12: 917-932.
KOUSKY VE \& KAYANO MT. 1994. Principal modes of outgoing Iongwave radiation and 250-mb circulation for the South American sector. J. Clim., 7: 1131-1143.

KUTZBACH JE. 1967. Empirical eigenvectors of sea-level pressure, surface temperature and precipitation complexes over North America. J. Applied Meteor., 6: 791-802.

LAU N-C \& NATH MJ. 2000. Impact of ENSO on the variability of the Asian-Australian monsoons as simulated in GCM experiments. J. Clim., 13: 4287-4309.

LIEBMANN B \& MARENGO JA. 2001. Interannual variability of the rainy season and rainfall in the Brazilian Amazon Basin. J Clim., 14: 43084318.

LIEBMANN B \& SMITH CA. 1996. A description of a complete (interpoled) outgoing longwave radiation dataset. Bull. Amer. Meteor. Soc., 77: 1275-1277.

LIEBMANN B, KILADIS GN, MARENGO JA, AMBRIZZI T \& GLICK JD. 1999. Submonthly convective variability over South America and the South Atlantic Convergence Zone. J. Clim., 12: 1877-1891. 

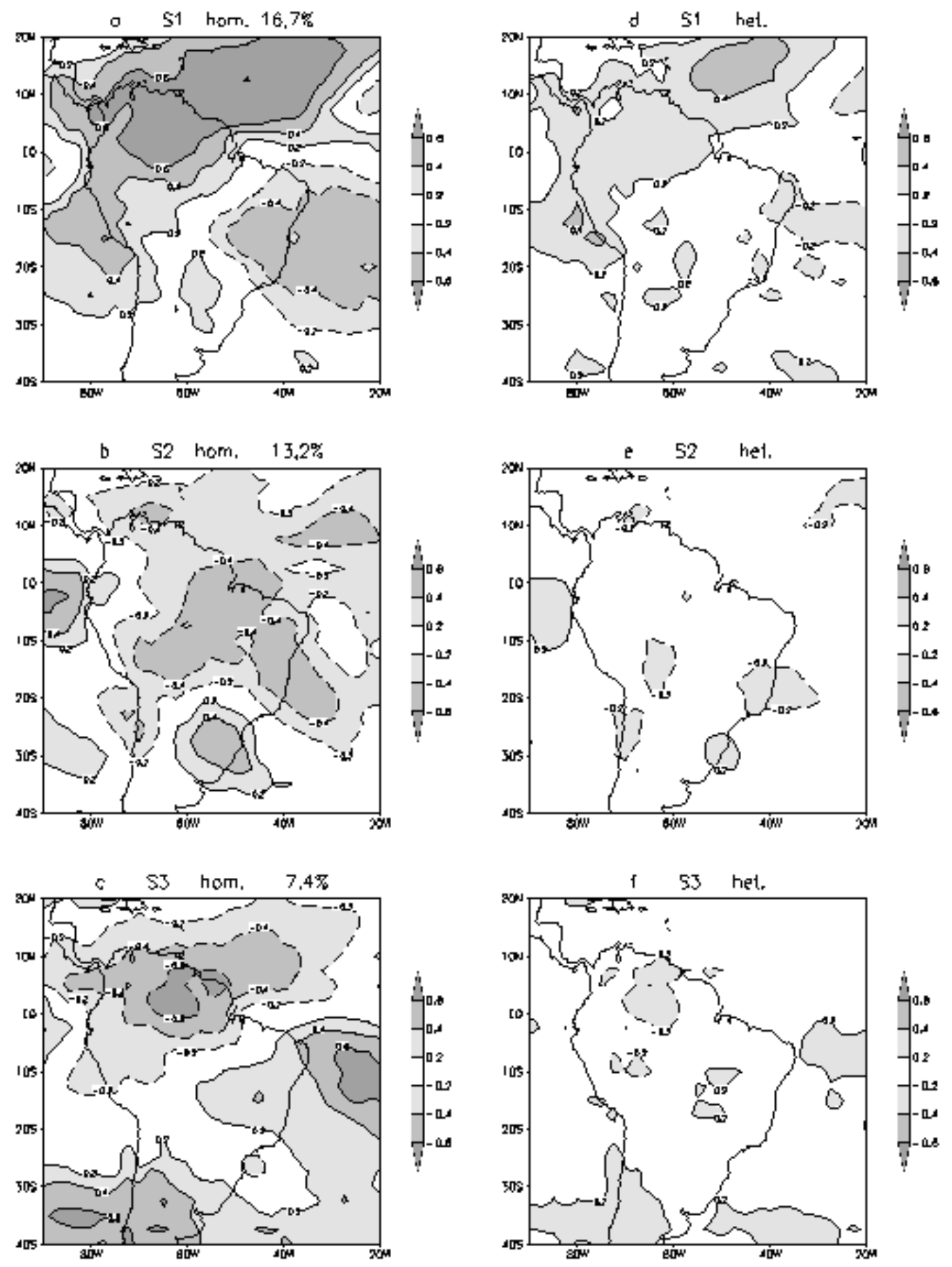

Figura 7 - Campos homogêneos (a, b, c) e heterogêneos (d, e, f) de ROL dos três primeiros modos da SVD entre as anomalias normalizadas de ROL sobre a América do Sul e de TSM sobre o Atlântico Norte para DJF de 1979/2000. Tom de cinza representa valores com nível de significância acima de $95 \%$ segundo $0 t$-Student's. 
a

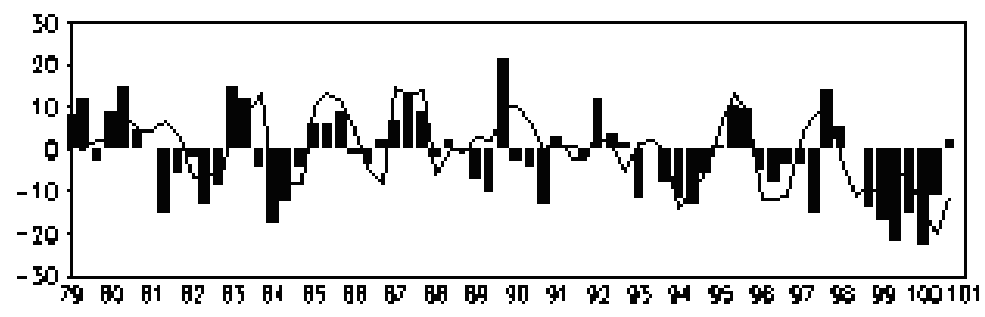

b

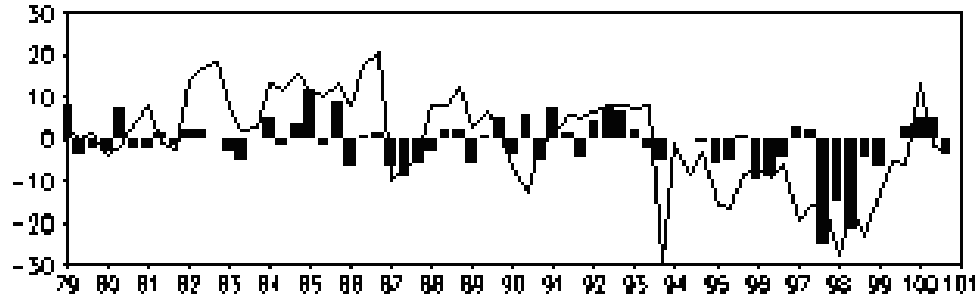

C

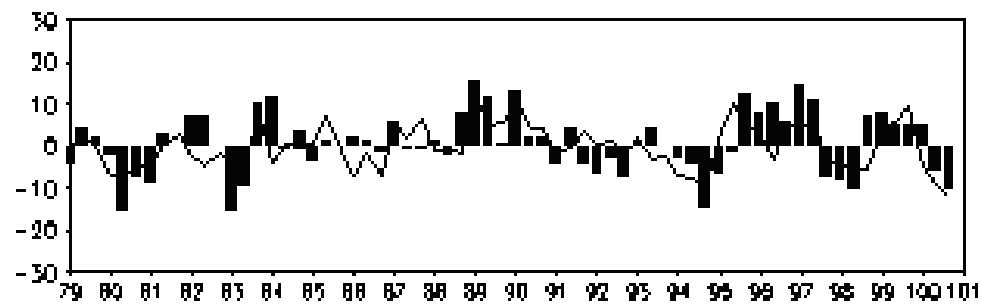

Figura 8 - Séries temporais dos três primeiros modos da SVD para as anomalias normalizadas de ROL (barras) sobre a América do Sul e de TSM (isolinhas) sobre o Atlântico Norte para DJF de 1979/2000. No eixo horizontal 100 e 101 representam os anos 2000 e 2001, respectivamente.

MANTUA NJ, HARE SR, ZHANG Y, WALLACE JM \& FRANCIS RA. 1997. Pacific Interdecadal Climate Oscillation with Impacts on Salmon Production. Bull. Amer. Meteor. Soc., 78: 1069-1079.

MOURA AD \& SHUKLA J. 1981. On the dynamics of droughts in Northeast Brazil: observations, theory and numerical experiments with a general circulation model. J. Atmos. Sci., 38: 2653-2675.

NOBRE P \& SHUKLA J. 1996. Variations of sea surface temperature, wind stress, and rainfall over the tropical Atlantic and South America. J. Clim., 9: 2464-2479.

NOGUÉS-PAEGLE J \& MO KC. 1997. Alternating wet and dry conditions over South America during summer. Mon. Wea. Rev, 125: 279-291.

PAEGLE JM \& MO KC. 2002. Linkages between Summer Rainfall Variability over South America and Sea Surface Temperature Anomalies. J. Climate, 15: 1389-1407.

PENG S \& ROBINSON WA. 2001. Relationships between atmospheric internal variability and the responses to an extratropical SST anomaly. J.
Clim., 14: 2943-2959.

RICHMAN MB. 1986. Rotation of principal components. J. Climatol., 6: 293-335.

ROBERTSON AW \& MECHOSO CR. 2000. Interannual and Interdecadal Variability of the South Atlantic Convergence Zone. Mon. Wea. Rev., 128: 2947-2957.

SARAVANAN R \& CHANG P. 2000. Interaction between Tropical Atlantic Variability and El Niño-Southern Oscillation. J. Clim. 13: 2177-2194.

TRENBERTH KE, STEPANIAK DP \& CARON JM. 2002. Interannual variations in the atmospheric heat budget. J. Geophys. Res., 107(D7): 1513715146.

VENEGAS SA, MYSAK LA \& STRAUB DN. 1997. Atmosphere -ocean coupled variability in the south Atlantic. J. Clim., 10: 2904-2920.

VON STORCH H \& NAVARRA A. 1999. Analysis of climate variability: applications of statistical techniques, Alemanha: Springer-Verlag, 403p. 
WALLACE JM, SMITH C \& BRETHERTON CS. 1992. Singular value decomposition of wintertime sea surface temperature and $500 \mathrm{mb}$ height anomalies. J. Clim., 5: 561-570.

WARD MN \& FOLLAND CK. 1991. Prediction of seasonal rainfall in the north nordeste of Brazil using eigenvectors of sea-surface temperature. Int. J. Climatol., 11: 711-743.

WILKS DS. 1995. Statistical Methods in the Atmospheric Sciences: an
Introduction, Cambrigde: Academic Press, 464 p.

WOLTER K. 1997. Trimming problems and remedies in COADS. J. Clim., 10: 1980-1997.

ZHANG C. 1993. Large-scale variability of atmosphere deep convection in relation to sea surface temperature in the tropics. J. Clim., 6: 18981913.

\section{NOTAS SOBRE O AUTOR}

Rosane R. Chaves. Meteorologista pelo Instituto de Astronomia, Geofísica e Ciências Atmosféricas (IAG) da USP. Mestrado e Doutorado em Meteorologia no INPE. Atualmente Professora Associada da Universidade Estadual do Norte Fluminense Darcy Ribeiro - UENF no Laboratório de Engenharia e Exploração de Petróleo - LENEP e Laboratório de Meteorologia - LAMET. 